\title{
Funny turns in Young badminton player: A case of cerebellar infarction secondary to vertebral artery dissection
}

\author{
Bhupendra Shah, Nawli Manandhar, Raju Paudel
}

Department of Neuroscience, Grande International hospital, Kathmandu, Nepal

Keywords: Cerebellar infarction, dizziness, stroke, vertebral artery dissection.

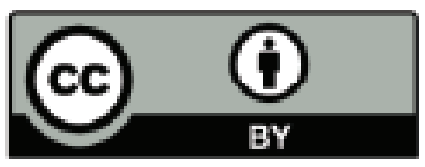

This work is licensed under a Creative Commons Attribution 4.0 Unported License.

\begin{abstract}
Spontaneous vertebral artery dissection is a rare cause of cerebellar infarction. Common presentations of cerebellar artery infarction are dizziness and ataxia. We are reporting a case of a 31-year-old male who presented with acute onset dizziness while playing badminton, who was diagnosed as cerebellar vermis infarction secondary to vertebral artery dissection.
\end{abstract}

\section{Introduction}

The spontaneous carotid artery and vertebral artery dissection caused $2 \%$ of all ischemic strokes.1 Common genetic causes of spontaneous vertebral dissection are Marfan syndrome, Ehler danlos syndrome, and autosomal dominant polycystic kidney disease, 2 whereas common environmental causes are events associated with neck extension like practicing yoga, painting ceiling, coughing, vomiting, sneezing, and different sports injuries. 3 Sports like scuba diving, combat sports, golf, basketball, and football are associated with vertebral artery dissection. 4

\section{*Corresponding Author:}

Dr Bhupendra Shah

Clinical fellow

Department of Neurosciences

Grande International hospital, Kathmandu, Nepal

Email: doctorbhupen@gmail.com 
Vertebral artery dissection while playing badminton is rarely reported in the literature, hence we are reporting a 31- year-old male who had cerebellar vermis infarction secondary to vertebral artery dissection that occurred while playing badminton.

\section{Case report}

A 31-year-old male was referred from the emergency department with a provisional diagnosis of Benign paroxysmal positional vertigo to the Outpatient Department of Neuroscience. The patient had complaints of dizziness for 3 days. The onset of dizziness occurred while playing badminton. It is a severe and rotatory type, continuously occurring in all positions. He also had difficulty walking due to dizziness and a tendency to fall towards the left side. There was associated history of nausea and 3 episodes of vomiting. The patient had a history of neck pain of mild to moderate in intensity aggravated on neck movement. $\mathrm{He}$ didn't have any history of headache, tinnitus, ear discharge, ear fullness, diplopia, and vision problem. He was diagnosed with a case of Type 2 Diabetes mellitus for 3 months and being treated with Metformin 500 milligram twice a day, Empaglifozin 10 milligram once daily, and Rosuvastatin 10 milligram once daily. He had no history of vertigo in the past.

On examination, the patient was conscious and cooperative. His blood pressure was 120/80 millimeters of mercury, respiratory rate was 18 cycles per minute, pulse rate was 80 beats per minute, regular, and temperature was 98 degrees Fahrenheit. His height was $170 \mathrm{~cm}$ and weight was $70 \mathrm{~kg}$ and his Body mass index was 24 $\mathrm{kg} / \mathrm{m} 2$. He had a wide-based ataxic gait. All other motors, sensory, and cranial examinations were within normal limits. Respiratory and cardiovascular system examination was normal. Baseline investigation of the patient was within normal limits. Magnetic resonance imaging(MRI) brain with stroke protocol (Figure 1) was done at first visit which demonstrated the cerebellar vermis infarction and Computed tomography (CT) angiography (Figure 2) showed narrowing of right vertebral artery lumen and intimal flap in the third segment.

We diagnosed him as the case of vertebral artery dissection with cerebellar vermis infarction. The patient was initially given tablet Aspirin 150 milligram once daily, Clopidogrel 75 milligram once daily which was changed to Rivaroxaban 20 milligram once daily and Rosuvastatin 20 milligram once daily. Dizziness of patient improved after seven days of treatment.

\section{Discussion}

We made of diagnosis of acute cerebellar vermis infarction secondary to right vertebral artery dissection based on the clinical history of acute onset dizziness with neck pain, examination finding of truncal ataxia, MRI head finding of a hyperintense lesion in cerebellar vermis in the diffusion-weighted image, and computed tomography angiography finding of the intimal flap in the vertebral artery.

Our patient had acute onset cerebellar infarction manifested with acute onset dizziness and associated neck pain. A similar case was reported by J Inamasu et al where a Young patient with cerebellar infarction that occurred secondary to vertebral artery dissection had associated neck pain.5 Presence of neck pain in a patient with acute dizziness might be a clue for vertebral artery dissection.

We detected vertebral dissection with the aid of CT angiography. It has the highest sensitivity (100\%) to detect vertebral artery dissection and common findings reported were arterial stenosis, arterial occlusion, aneurysm, double lumen, and intimal flap.6 Our patient had an intimal flap and vertebral artery narrowing in CT angiography. On suspicion of vertebral artery dissection,
CT angiography is the choice of investigation of lesion detection.

Initially, we treated the patient with dual antiplatelet Aspirin and Clopidogrel which was later changed to a novel anticoagulant. As reported by Dauo B et al, the rate of new or recurrent events was similar in dual antiplatelet and anticoagulants treatment in treating patients with vertebral artery dissection. 7 We treated our patient with a novel anticoagulant as per the patient preference.

Conclusion: Cerebellar infarction secondary to vertebral artery dissection is one of the causes of acute onset dizziness among sportsmen. Timely diagnoses and treatment prevent the lifethreatening complication of the condition.

Acknowledgment: We would like to acknowledge the Patient giving us consent to write this case report.

\section{References}

1. Bassetti C, Carruzzo A, Sturzenegger M,et al. Recurrence of cervical artery dissection. A prospective study of 81 patients. Stroke. 1996;27:1804-1807.

2. Schievink WI, Michels VV, Piepgras DG. Neurovascular manifestations of heritable connective tissue disorders. A review. Stroke. 1994;25(4):889-903.

3. Fisher CM, Ojemann RG, Roberson GH. Spontaneous dissection of cervico-cerebral arteries. Can J Neurol Sci. 1978;5:9-19.

4. Schlemm L, Nolte CH, Engelter ST, et al. Cervical artery dissection after sports-An analytical evaluation of 190 published cases. Eur Stroke J. 2017;2(4):335-345.

5. Inamasu J, Nakae S, Kato Y, et al. Clinical Characteristics of Cerebellar Infarction Due to Arterial Dissection. Asian J Neurosurg. 2018;13(4):995-1000.

6. Gottesman RF, Sharma P, Robinson KA et al. Imaging characteristics of symptomatic vertebral artery dissection: a systematic review. Neurologist. 2012;18(5):255-260.

7. Daou B, Hammer C, Mouchtouris N, et al. Anticoagulation vs antiplatelet treatment in patients with carotid and vertebral artery dissection: A study of 370 patients and literature review. Neurosurgery. 2017;80(3):368-379. 


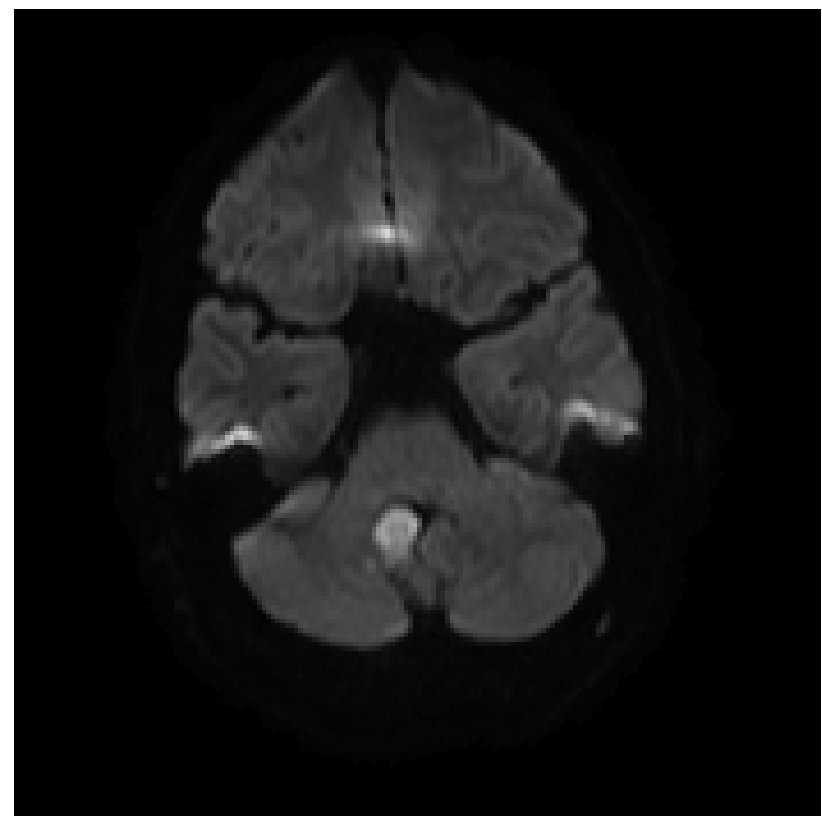

Figure 1: Diffusion weighted image of Patient showing diffusion restriction at vermis of cerbellum

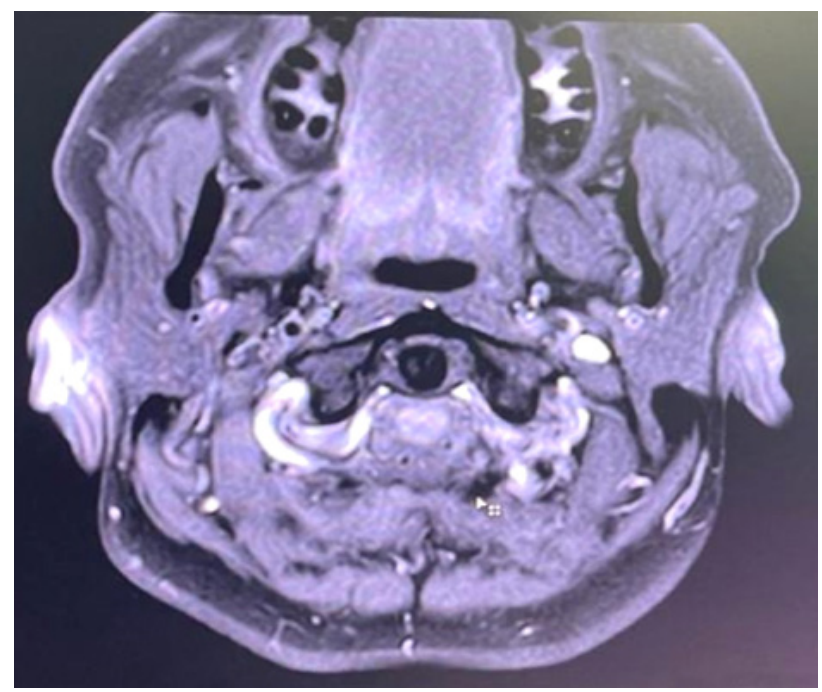

Figure 2: Figure 2 Showing intimal flap in right vertebral artery as shown by arrow. 